\title{
Efficacy and Safety of VNS Therapy or Continued Medication Management for Treatment of Adults with Drug-Resistant Epilepsy: Systematic Review and Meta-Analysis
}

Joan Conry

Sarah Batson (D Sarah.batson@mtechaccess.co.uk)

Stephen Mitchell

Rohit Shankar

Jane Boggs

Rodney Radtke

Francesca Barion

Joanna Murphy

Vanessa Danielson

Systematic Review

Keywords: Anti-seizure medication, VNS Therapy, Meta-analysis, Drug-resistant epilepsy, Seizure frequency

Posted Date: December 17th, 2021

DOI: https://doi.org/10.21203/rs.3.rs-1169679/v1

License: (c) (i) This work is licensed under a Creative Commons Attribution 4.0 International License.

Read Full License 


\title{
Efficacy and Safety of VNS Therapy or Continued Medication \\ Management for Treatment of Adults with Drug-Resistant Epilepsy: Systematic Review and Meta-Analysis
}

Sarah Batson, PhD, ${ }^{a}$ Rohit Shankar, FRCPsych, ${ }^{b}$ Joan Conry, MD, ${ }^{c}$ Jane Boggs, MD, ${ }^{d}$ Rodney Radtke, MD, ${ }^{e}$ Stephen Mitchell, PhD, ${ }^{a}$ Francesca Barion, PhD, ${ }^{f}$ Joanna Murphy, ${ }^{f}$ Vanessa Danielson, $\mathrm{MSc}^{\mathrm{g}}$

\author{
Affiliations: \\ ${ }^{a}$ Mtech Access Limited, United Kingdom \\ ${ }^{\mathrm{b}}$ Neuropsychiatry, Peninsula School of Medicine, University of Plymouth, United Kingdom \\ ' Neurology, Children's National, Washington, District of Columbia, USA \\ ${ }^{d}$ Neurology, Wake Forest Baptist Health, Winston-Salem, North Carolina, USA \\ e Duke University School of Medicine, Durham, North Carolina, USA \\ ${ }^{f}$ Pricing, Health Economics, Market Access and Reimbursement (PHEMAR), LivaNova, Europe \\ ${ }^{g}$ Global VP, PHEMAR, LivaNova, Europe
}

ORCID:

RS: 0000-0002-1183-6933

JC: 0000-0002-1397-1370

JB: 0000-0003-1083-2954

RR: 0000-0001-5029-5829

SB, SM, FN, JM, VD: NA

Corresponding author:

Sarah Batson, Mtech Access Limited, 30 Murdock Road, Bicester, Oxfordshire, OX26 4PP, UK Email: sarah.batson@mtechaccess.co.uk 


\begin{abstract}
:
Vagus nerve stimulation (VNS) Therapy ${ }^{\circ}$ is an adjunctive neurostimulation treatment for people with drug-resistant epilepsy (DRE) who are unwilling to undergo resective surgery, have had unsuccessful surgery or are unsuitable for surgery. A systematic review and meta-analysis were conducted to determine the treatment effects of VNS Therapy as an adjunct to anti-seizure medications (ASMs) for the management of adults with DRE.
\end{abstract}

A literature search was performed in August 2020 of the Medline ${ }^{\circledR}$, Medline ${ }^{\circledR}$ Epub Ahead of Print, Embase, and the Cochrane library databases. Outcomes examined included reduction in seizure frequency, seizure freedom, ASM load, discontinuations, and serious adverse events (SAEs). Comparators included best medical practice, ASMs, low-stimulation or sham VNS Therapy.

Four RCTs and six comparative observational studies were identified for inclusion. Against comparators, individuals treated with VNS had a significantly better odds of experiencing a $\geq 50 \%$ reduction in seizure frequency (OR: 2.27 [95\% Cl: 1.47, 3.51]; $p=0.0002$ ), a $\geq 75 \%$ reduction in seizure frequency (OR: 3.56 [95\% Cl: 1.59, 7.98]; $\mathrm{p}=0.002$ ) and a reduced risk for increased ASM load (risk ratio: 0.36 [95\% Cl: 0.21, 0.62 ]; $p=0.0002$ ). There was no difference in the odds of discontinuation or the rate of SAEs between VNS versus comparators.

This meta-analysis demonstrated the benefits of VNS Therapy in people with DRE, which included improvement in seizure frequency without an increase in the rate of SAEs or discontinuations, thereby supporting the consideration of VNS Therapy for people who are not responding to ASMs and those unsuitable or unwilling to undergo surgery.

\title{
Key words:
}

Anti-seizure medication

VNS Therapy

Meta-analysis

Drug-resistant epilepsy

Seizure frequency 


\section{Statements and Declarations}

\section{Conflict of interest/Competing interests:}

Rohit Shankar has received institutional and research support from LivaNova, UCB, Eisai, Veriton Pharma, Bial, Averelle and GW pharmaceuticals outside the submitted work. He was the author/lead of the Royal College of Psychiatrists reports CR203, CR206 and the National Step Together Report. He is also the medical lead of the freely available SUDEP and Seizure safety Checklist and EpSMon App. He is the developmental disabilities representative in the recent NICE revision/update of the epilepsies (2022), the NHS RightCare report (2020), the NHS England specialist commissioning report for epilepsy (2021) and the National Confidential Inquiry into epilepsy deaths (2021-22).

Joan Conry has received research funding from LivaNova.

Jane Boggs has received research funding from LivaNova, Medtronic and UCB.

Francesca Barion, Joanna Murphy and Vanessa Danielson are employees of LivaNova.

Sarah Batson, Stephen Mitchell and Rodney Radtke have no competing interests to declare that are relevant to the content of this article.

\section{Funding:}

This study was funded by LivaNova.

\section{Ethical standards:}

The manuscript does not contain clinical studies or patient data.

\section{Acknowledgements:}

Christa Zachariah and Shauna Garris, Medical Science Liaison Managers at LivaNova for their contribution to the tabulation of the data.

Writing support was provided by Éanna Connaughton (Mtech Access).

\section{Author contributions:}

Francesca Barion, Joanna Murphy and Vanessa Danielson conceptualised the project. Sarah Batson and Stephen Mitchell planned and conducted the investigation and the formal analysis. All authors were involved in the writing, review and editing of the manuscript. 


\section{INTRODUCTION}

Epilepsy is a common neurological condition, affecting approximately 50 million people globally [1]. At least 30\% exhibit drug-resistant epilepsy (DRE) and continue to suffer seizures despite treatment [2]. DRE is defined by the International League Against Epilepsy (ILAE) as failure of adequate trials of two tolerated, appropriately chosen and used anti-seizure medication (ASM) schedules (whether as monotherapies or in combination) to achieve sustained seizure freedom [3].

People with DRE experience significantly more comorbidities, including depression, seizure-related injuries, and neurological deficits than those without epilepsy or with epilepsy that responds to treatment $[4,5]$, and have significantly higher mortality risk [6, 7]. DRE is also associated with sudden unexplained death in epilepsy (SUDEP) which represents a major cause of death in this population [810]. People with DRE have substantially higher healthcare costs than those who are seizure-free, including costs associated with medical investigations, treatment costs, emergency room visits, hospitalisations, and outpatient care [18-20]. In addition, people with DRE frequently report feeling stigmatised because of their epilepsy [11].

It has been reported that seizures in $>30 \%$ of people with DRE remain uncontrolled and this statistic has not changed over the last 20 years despite the availability of new ASMs [12, 13]. For people who fail to experience sufficient seizure reduction with pharmacologic therapy, alternative approaches include epilepsy brain surgery [14], diet modification [15], and neurostimulation devices [16-19], including Vagus Nerve Stimulation Therapy ${ }^{\circledR}\left(\right.$ VNS Therapy $\left.{ }^{\circledR}\right)[20,21]$.

While for many people with DRE brain surgery can be curative and result in seizure freedom, with up to $52 \%$ of people remaining seizure-free (apart from simple partial seizures) 5 years post-intervention [22, 23]. However, not all individuals are suitable candidates, and uptake of surgery is limited by hesitancy, in part due to fears of postoperative permanent neurological deficits [24].

VNS Therapy represents a commonly used neurostimulation option for people with DRE who do not wish to undergo cranial surgery or laser interstitial thermal ablation, who have had unsuccessful surgery or are not suitable for surgery (including individuals with intellectual impairment who may be unable to understand and consent to a non-reversible procedure) [25-27]. VNS is a minimally invasive extracranial device which delivers mild, intermittent electrical pulses to the vagus nerve which then stimulates areas in the brain responsible for seizures $[28,29]$. This results in a reduction in seizure frequency $[20,21]$. VNS Therapy ${ }^{\oplus}$ has been in clinical use in Europe since 1994 [30] and in the USA since 1997 [31]. 
This systematic literature review (SLR) and meta-analysis examined the treatment effects of VNS Therapy at up to 2 years as an adjunct to ASMs for the management of adults with DRE based on the most up-to-date evidence from randomised controlled trials (RCTs) and comparative observational studies. 


\section{MATERIALS AND METHODS}

\subsection{SLR}

An SLR was conducted on the 25th of August 2020 (in alignment with the Preferred Reporting Items for Systematic reviews and Meta-Analyses [PRISMA] checklist) [32] to identify relevant clinical studies (RCTs and observational comparative studies) comparing VNS Therapy as an adjunct to ASMs with relevant comparator arms in adults with DRE followed by a meta-analysis to determine treatment effects for several efficacy and safety outcomes.

The SLR searched the electronic databases of Medline $^{\circledR}$, Medline $^{\circledR}$ Epub Ahead of Print (In-Process \& Other Non-Indexed Citations), Embase, and the Cochrane library to identify relevant clinical studies (RCTs, controlled clinical studies, and prospective registries) examining VNS Therapy and other interventions of interest for the management of patients with DRE. Additional searches of congress proceedings from the past 3 years (American Epilepsy Society [AES], Congress of Neurological Surgeons [CNS] Annual Meeting, European Congress on Epileptology [ECE], International Epilepsy Congress [IEC], International Neuromodulation Society [INS] Congress), reference lists of included publications, and Health Technology Assessment (HTA) bodies were conducted to identify relevant evidence. Search terms are listed in the Supplementary Materials. Citations were screened by a single analyst and independently checked by a second analyst; any discrepancies were resolved by consensus. Outcome data were extracted to a Microsoft ${ }^{\circledR}$ Excel spreadsheet.

For this analysis, the eligibility criteria included comparative clinical studies of VNS Therapy for the management of DRE conducted predominantly in an adult population (i.e. $>50 \%$ of individuals were aged $\geq 18$ years). Eligible comparators to VNS Therapy were: (1) best medical practice (BMP), (2) continuation of stable ASM regimen, (3) addition of ASM, and (4) low-stimulation VNS Therapy (parameters defined in Table 3).

\section{Data collection and risk of bias assessment}

General patient/participant demographics were extracted such as age at time of implant, sex, type of seizure and baseline seizure frequency. Outcomes of interest included reduction in seizure frequency, seizure freedom, ASM load, discontinuations, and serious adverse events (SAEs).

Quality (risk of bias) assessment of RCTs was conducted using the seven-criteria checklist provided in Section 2.5 of the National Institute for Health and Care Excellence (NICE) single technology appraisal 
(STA) user guide for RCTs [33]. Observational studies were assessed using the quality assessment tool for quantitative studies of the Effective Public Health Practice Project (EPHPP) [34].

\subsection{Meta-analysis}

Evidence synthesis was conducted via pair-wise meta-analyses based on RCT and comparative observational studies. While observational comparative evidence is of lower quality compared with RCTs due to the inherent bias within such studies, their inclusion was deemed appropriate as observational comparative studies provide longer follow-up compared with RCTs. Pair-wise meta-analyses were conducted for the outcomes of interest previously described. For one RCT (PuLsE) [35], which reported outcomes up to 2-years post-surgery, the outcome results were restricted to the 12-month time point. The 12-months results were included in the meta-analysis. Outcomes for this study were restricted to 12 months in order to facilitate data comparisons as all other RCTs included in the meta-analysis had shorter follow ups (range: 3.5-6 months).

\subsection{Statistical analysis}

Evidence synthesis was conducted via pair-wise meta-analyses based on RCT and comparative observational studies where available. The pair-wise meta-analyses were conducted in RevMan 5.3. Heterogeneity was assessed using the chi-squared and I-squared statistics. Results were presented as an odds ratio (OR) or weighted mean difference with $95 \%$ confidence intervals ( $\mathrm{Cls}$ ). 


\section{RESULTS}

\subsection{SLR}

\section{RCTs and comparative observational studies}

A total of 48 publications (on 30 unique studies, see Supplementary Materials Table 1) were identified for potential inclusion. In total, 38 VNS Therapy studies did not meet eligibility criteria for inclusion in this meta-analysis (due to publications including non-relevant comparators, no outcomes of interest or publications were superseded by a linked publication); 10 studies were identified for inclusion (four RCT studies [4 unique publications] and six comparative observational studies [5 unique publications]) (Fig. 1Fig.). The publication dates ranged between 1993 and 2015.

Four primary study publications from four RCTs in an adult population were included in the analysis; 13 publications linked to these RCTs were excluded as they did not report outcomes of interest or were superseded by the primary study publication [36-47]. A single RCT study (with two linked publications; see Supplementary Materials Table 1) was excluded due to unclear reporting of the enrolled population (i.e. proportion of adults) $[48,49]$. A total of 12 adult comparative observational study publications were excluded for the following reasons: no comparator $(n=4)$ [50-53], population of interest $(n=2)[54,55]$, reported no outcomes of interest $(n=5)[56-60]$ and superseded by a linked primary publication $(n=1)[61]$.

\subsection{Study and participant characteristics}

Study design and baseline participant characteristics of the VNS Therapy studies are shown in Table 1 and Table 2Table. The study duration ranged from 3-4.5 months for the majority of RCTs $[20,21,62]$, with one RCT study lasting 24 months [35]. The study duration for comparative observational studies was typically $>12$ months (range: 3-32 months) [63-68]. Where reported, the mean participant age ranged 32-41 years for the RCT studies $[20,21,35,62]$ and $25-40$ years for comparative observational studies. Disease duration was only reported by half of the studies included $(n=5)$, with the mean duration ranging between $20-23$ years for RCT studies $[20,21]$ and $17-26$ years for comparative observational studies $[63,66,68]$. Mean seizure frequency ranged from 0.6-1.7 and 0.1-3.5 seizures per day for RCT and non-comparative observational studies, respectively. The mean number of drugs used ranged between $2-3$ for both $\mathrm{RCT}[20,21,35]$ and comparative observational studies $[64-66,68]$. Three studies compared a low-stimulation setting (control arm) plus background ASMs with a high-stimulation setting; the difference between the low- and high-stimulation parameters is provided in Table 3. 
Rationale for using the low-stimulation included the facilitation of titration, ethical reasons, inclusion of an active control group and to permit a double-blind trial design $[20,62,69]$. The majority of studies $(n=7)$ compared VNS Therapy with a continuation of the participants' current ASM regimen; only one comparative observational study reported the type of ASMs participants were taking [65]. None of the included studies made a specific comparison between VNS Therapy and the latest generation of ASMs (e.g. those licensed in the last two decades [i.e. lacosamide, cannabidiol, brivaracetam, perampanel etc.]). 


\section{Table 1: Summary of included RCTs}

21

\begin{tabular}{|c|c|c|c|c|c|c|c|c|c|c|c|c|}
\hline $\begin{array}{l}2 \text { 2Author / } \\
23 \text { Publication } \\
24 \\
25\end{array}$ & Location & $\begin{array}{l}\text { Treatment } \\
\text { length }\end{array}$ & $\begin{array}{l}\text { Duration of } \\
\text { follow-up }\end{array}$ & Comparators & $\begin{array}{l}\text { Sample } \\
\text { size, } n\end{array}$ & $\begin{array}{l}\text { Age at implant } \\
\text { mean (SD } \\
\text { [range]) }\end{array}$ & $\begin{array}{l}\text { Sex, F } \\
\text { (n [\%]) }\end{array}$ & $\begin{array}{l}\text { Mean } \\
\text { duration of } \\
\text { disease, } \\
\text { years }\end{array}$ & $\begin{array}{l}\text { Mean no. of } \\
\text { ASMs used at } \\
\text { baseline }\end{array}$ & Seizure types, ${ }^{\dagger} n\left(\%^{\ddagger}\right)$ & Seizure frequency & Outcomes Assessed \\
\hline \multirow{2}{*}{$\begin{array}{l}2 \text { Ryvlin } \\
277^{2014}[35] \\
28 \text { (PuLsE) } \\
29 \\
30 \\
31 \\
32\end{array}$} & \multirow[t]{2}{*}{$\begin{array}{l}\text { Canada, } \\
\text { Europe }\end{array}$} & \multirow[t]{2}{*}{2 years } & \multirow[t]{2}{*}{2 years } & VNS + BMP & 54 & $38 \pm 13$ & $24(50)$ & \multirow[t]{2}{*}{ NR } & $\begin{array}{l}3.5 \text { (SD: } \\
\pm 1.17)\end{array}$ & $\begin{array}{l}\text { Structural/metabolic: } 26 \\
\text { (54) Unknown: } 22 \text { (46) }\end{array}$ & $\begin{array}{l}\text { Median: } 5 \text { per } \\
\text { week (range: } 1 \\
\text { to } 123 \text { ) }\end{array}$ & \multirow{2}{*}{$\begin{array}{l}\text { Seizure frequency } \\
\text { (including } \geq 50 \% \text { ) } \\
\text { HRQoL } \\
\text { ASM usage } \\
\text { Adverse events }\end{array}$} \\
\hline & & & & BMP (ASM) & 58 & $41 \pm 11$ & $21(44)$ & & $\begin{array}{l}3.2 \text { (SD: } \\
\pm 1.22)\end{array}$ & $\begin{array}{l}\text { Structural/metabolic: } 26 \\
\text { (54) Unknown: } 22 \text { (46) }\end{array}$ & $\begin{array}{l}\text { Median: } 4 \text { per } \\
\text { week (range: } 1 \\
\text { to } 42 \text { ) }\end{array}$ & \\
\hline \multirow{2}{*}{ 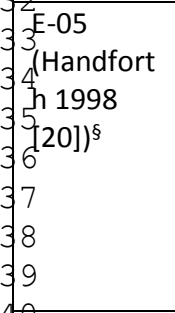 } & \multirow[t]{2}{*}{ US } & \multirow[t]{2}{*}{3 months } & \multirow[t]{2}{*}{$\begin{array}{l}3.5-4 \\
\text { months }\end{array}$} & $\begin{array}{l}\text { VNS high } \\
\text { stim }\end{array}$ & 95 & $\begin{array}{l}32.1(10.8 \\
[13-54])\end{array}$ & $\begin{array}{l}46 \\
(48.4)\end{array}$ & \multirow[t]{2}{*}{23} & $2.2(\mathrm{SD}: \pm 0.7)$ & \multirow[t]{2}{*}{$\begin{array}{l}\text { CPS or partial + } \\
\text { secondarily generalised, } \\
+/ \text { - other seizure types }\end{array}$} & $\begin{array}{l}\text { Mean: } 1.59 \text { per } \\
\text { day (SD } \pm 1.96) \\
\text { Median: } 0.58 \\
\text { per day }\end{array}$ & \multirow{2}{*}{$\begin{array}{l}\text { Seizure frequency } \\
\text { (including } \geq 50 \% \text {, } \\
\geq 75 \% \text { and seizure } \\
\text { free) } \\
\text { ASM usage } \\
\text { Quality of life } \\
\text { Adverse events } \\
\text { Discontinuations }\end{array}$} \\
\hline & & & & $\begin{array}{l}\text { VNS low } \\
\text { stim }\end{array}$ & 103 & $\begin{array}{l}34.2(10.1 \\
[15-60])\end{array}$ & $\begin{array}{l}59 \\
(57.3)\end{array}$ & & $2.1(\mathrm{SD}: \pm 0.7)$ & & $\begin{array}{l}\text { Mean: } 0.97 \text { per } \\
\text { day (SD } \pm 0.94 \text { ) } \\
\text { Median: } 0.51 \\
\text { per day }\end{array}$ & \\
\hline $\begin{array}{l}4 I_{\text {E-03 }}(\text { Salinsky } \\
421995[21]) \\
43 \\
44 \\
45 \\
46 \\
47\end{array}$ & $\begin{array}{l}\text { Europe, } \\
\text { North } \\
\text { America }\end{array}$ & 3 months & $\begin{array}{l}3.5-4 \\
\text { months }\end{array}$ & $\begin{array}{l}\text { VNS high } \\
\text { stim }\end{array}$ & 54 & 33.1 (NR) & $21(39)$ & 23.1 & 2.09 & $\begin{array}{l}\text { SPS: } 24 \text { (44.4\%) } \\
\text { CPS: } 50 \text { (92.6\%) } \\
\text { Partial secondarily } \\
\text { generalised: } 38 \text { (70.4\%) }\end{array}$ & $\begin{array}{l}\text { Mean } 1.49 \text { per } \\
\text { day (SD: NR) } \\
\text { Median: } 0.73 \\
\text { per day }\end{array}$ & $\begin{array}{l}\text { Seizure frequency } \\
\text { (including } \geq 50 \%, \\
\geq 75 \% \text { and seizure } \\
\text { free) } \\
\text { Adverse events }\end{array}$ \\
\hline $\begin{array}{l}48 \text { Landy } \\
491993[62] \\
50 \\
51 \\
52 \\
53 \\
54 \\
5\end{array}$ & US & $\begin{array}{l}3-4.5 \\
\text { months }\end{array}$ & $\begin{array}{l}6.5 \\
\text { months }\end{array}$ & $\begin{array}{l}\text { VNS high } \\
\text { stim }\end{array}$ & 4 & NR & NR & NR & NR & NR & NR & $\begin{array}{l}\text { Seizure frequency } \\
\text { Adverse events }\end{array}$ \\
\hline
\end{tabular}

5 Abbreviations: ASM, anti-seizure medication; BMP, best medical practice; CPS, complex partial seizures; HRQoL, health-related quality of life; NR, not reported; RCT, randomised controlled trial; SD, standard 5 deviation; SPS, simple partial seizures; VNS, Vagus Nerve Stimulation.

58 59 May be counted in more than one type; $¥$ Where applicable; § Cyberonics-sponsored VNS study (Cyberonics is owned by LivaNova).

60

61

62

63 


\begin{tabular}{|c|c|c|c|c|c|c|c|c|c|c|c|c|}
\hline \\
\hline \multirow{7}{*}{\multicolumn{13}{|c|}{$\begin{array}{l}14 \\
15 \\
16 \\
17 \\
18 \\
19 \\
\text { Tâble 2: Summary of included comparative observational studies } \\
21\end{array}$}} \\
\hline & & & & & & & & & & & & \\
\hline & & & & & & & & & & & & \\
\hline & & & & & & & & & & & & \\
\hline & & & & & & & & & & & & \\
\hline & & & & & & & & & & & & \\
\hline & & & & & & & & & & & & \\
\hline $\begin{array}{l}\text { 2Aputhor / } \\
\text { 2Pyblication } \\
24 \\
25\end{array}$ & Location & \begin{tabular}{|l|} 
Treatment \\
length
\end{tabular} & Comparators & $\begin{array}{l}\text { Duration of } \\
\text { Follow-up }\end{array}$ & $\begin{array}{l}\text { Sample } \\
\text { size, } n\end{array}$ & $\begin{array}{l}\text { Age at implant } \\
\text { mean (SD } \\
\text { [range]) }\end{array}$ & $\begin{array}{l}\text { Sex, F } \\
\text { (n [\%]) }\end{array}$ & $\begin{array}{l}\text { Mean } \\
\text { duration } \\
\text { of } \\
\text { disease }\end{array}$ & $\begin{array}{l}\text { Mean no. } \\
\text { of ASMs } \\
\text { used }\end{array}$ & Seizure types, ${ }^{+} n\left(\%^{\ddagger}\right)$ & $\begin{array}{l}\text { Seizure } \\
\text { frequency }\end{array}$ & $\begin{array}{l}\text { Outcomes } \\
\text { Assessed }\end{array}$ \\
\hline \multirow{2}{*}{$\begin{array}{l}25 \\
2 \text { gonen } 2015 \\
2[\text { [94] } \\
2 \text { (Gomparative } \\
2 \text { rgtrospective } \\
3 \text { goservational } \\
2^{\text {study) }}\end{array}$} & \multirow[t]{2}{*}{ Israel } & \multirow[t]{2}{*}{$\geq 1$ year } & VNS + ASM & 5.67 years & 33 & \multirow[t]{2}{*}{$\begin{array}{l}>18 \text { years of } \\
\text { age }\end{array}$} & $\begin{array}{l}14 \\
(42.4)\end{array}$ & \multirow[t]{2}{*}{ NR } & $\begin{array}{l}2.91 \text { (SD: } \\
\pm 0.95)\end{array}$ & \multirow[t]{2}{*}{ NR } & $\begin{array}{l}\text { Mean: } 3.52 \\
\text { per day (SD } \\
0.67)\end{array}$ & \multirow[t]{2}{*}{$\begin{array}{l}\text { Seizure } \\
\text { frequency, ASM } \\
\text { usage }\end{array}$} \\
\hline & & & ASM & 4.04 years & 47 & & $\begin{array}{l}26 \\
(55.3)\end{array}$ & & $\begin{array}{l}2.32 \text { (SD: } \\
\pm 0.98)\end{array}$ & & $\begin{array}{l}\text { Mean: } 3.15 \\
\text { per day (SD } \\
0.72 \text { ) }\end{array}$ & \\
\hline \multirow{2}{*}{$\begin{array}{l}3 \text { Hoppe } 2013 \\
3[\text { [6] } \\
3 \text { (Comparative, } \\
344 \text { Case control)§ } \\
35 \\
36 \\
37 \\
38\end{array}$} & \multirow[t]{2}{*}{$\begin{array}{l}\text { German } \\
y\end{array}$} & \multirow[t]{2}{*}{$>2$ years } & $\begin{array}{l}\text { VNS + BMP } \\
\text { (ASM) }\end{array}$ & \multirow[t]{2}{*}{$\begin{array}{l}6.8 \text { yrs (SD } \\
2.1 \text {, range } 2- \\
13)\end{array}$} & 20 & 39.8 (SD: 10.2) & $8(40)$ & $\begin{array}{l}25.7 \\
\text { (SD: } \\
13.4) \\
\end{array}$ & $\begin{array}{l}2.47 \text { (SD: } \\
\pm 0.77)\end{array}$ & $\begin{array}{l}\text { SPS: } 8(40 \%) \\
\text { CPS: } 18(90 \%) \\
\text { SGS: } 10(50 \%) \\
\end{array}$ & $\begin{array}{l}\text { Mean: } 68.4 \\
\text { (SD 206.3) per } \\
\text { month }\end{array}$ & \multirow{2}{*}{$\begin{array}{l}\text { Seizure } \\
\text { frequency, ASM } \\
\text { use and } \\
\text { tolerability, VNS } \\
\text { tolerability } \\
\text { HRQoL, ASM } \\
\text { use, adverse } \\
\text { events }\end{array}$} \\
\hline & & & BMP (ASM) & & 20 & 39 (SD: 8.5) & $8(40)$ & $\begin{array}{l}21.0 \\
\text { (SD: 9.2) }\end{array}$ & $\begin{array}{l}2.24 \text { (SD: } \\
\pm 0.44)\end{array}$ & $\begin{array}{l}\text { SPS: } 7(35 \%) \\
\text { CPD: } 17(85 \%) \\
\text { SPS: } 12(60 \%)\end{array}$ & $\begin{array}{l}\text { Mean } 8.2 \text { (SD } \\
\text { 10.4) per } \\
\text { month }\end{array}$ & \\
\hline \multirow{2}{*}{$\begin{array}{l}\text { 39yarrosu } 2003 \\
4[65] \\
4 \text { (Eomparative } \\
4 \text { Prospective } \\
43 \text { case control) } \\
44 \\
45 \\
46\end{array}$} & \multirow[t]{2}{*}{ Italy } & \multirow[t]{2}{*}{1 year } & VNS implant & \multirow[t]{2}{*}{1 year } & 10 & $33.1(23-44)$ & $4(40)$ & \multirow[t]{2}{*}{ NR } & 1.9 & \multirow[t]{2}{*}{ CPS } & $\begin{array}{l}\text { Mean } 156 \\
\text { (range: } 98- \\
\text { 212) per } \\
\text { trimester }\end{array}$ & \multirow[t]{2}{*}{$\begin{array}{l}\text { Seizure } \\
\text { frequency, } \\
\text { GRD } \\
\text { distribution }\end{array}$} \\
\hline & & & No implant & & 7 & $30.8(21-42)$ & $3(43)$ & & 1.9 & & $\begin{array}{l}\text { Mean } 150 \\
\text { (range 88- } \\
\text { 206) per } \\
\text { trimester }\end{array}$ & \\
\hline \multirow{3}{*}{$\begin{array}{l}\text { 4'Boon } 2002 \\
4[83] \\
4 \text { (Comparative } \\
5 \text { Prospective } \\
5 \text { Cohort) } \\
52 \\
53 \\
54 \\
55 \\
56 \\
57 \\
58 \\
59\end{array}$} & \multirow[t]{3}{*}{ Belgium } & \multirow[t]{3}{*}{$\begin{array}{l}\text { Average } 26 \\
\text { months } \\
\text { [range 12- } \\
57 \text { months] }\end{array}$} & VNS + ASM & $\begin{array}{l}29 \text { months } \\
\text { (range: } 12- \\
57)\end{array}$ & 25 & $\begin{array}{l}31 \text { years } \\
\text { (range: } 12-49 \\
\text { years) }\end{array}$ & \multirow[t]{3}{*}{ NR } & \multirow[t]{3}{*}{$\begin{array}{l}21 \text { years } \\
\text { (range: } \\
2-50 \\
\text { years) }\end{array}$} & \multirow[t]{3}{*}{ NR } & $\begin{array}{l}\text { SPS + SG: } 15 \text { (60\%) } \\
\text { CPS + SG/SPS: } 3(12 \%) \\
\text { CPS + SG/atonic: } 2(8 \%) \\
\text { CPS: } 5 \text { (20\%) }\end{array}$ & $\begin{array}{l}\text { Mean: } 21 \text { per } \\
\text { month (range: } \\
2 \text { to } 180 \text { ) }\end{array}$ & \multirow{3}{*}{$\begin{array}{l}\text { Epilepsy-related } \\
\text { direct medical } \\
\text { costs } \\
\text { Seizure } \\
\text { frequency } \\
\text { Number and } \\
\text { dosage of ASMs } \\
\text { Number of } \\
\text { hospital } \\
\text { admission days } \\
\text { clinic visits and } \\
\text { laboratory tests }\end{array}$} \\
\hline & & & Surgery & $\begin{array}{l}28 \text { months } \\
\text { (range, 12- } \\
54 \text { ) }\end{array}$ & 35 & $\begin{array}{l}32 \text { (range: } 10- \\
60 \text { years) }\end{array}$ & & & & $\begin{array}{l}\text { SPS + SG: } 1(3 \%) \\
\text { CPS + SG: } 17(49 \%) \\
\text { CPS: } 14(40 \%) \\
\text { GTC: } 1(3 \%) \\
\text { CPS/atonic: } 1(3 \%) \\
\text { CPS + SG/atonic: } 1(3 \%)\end{array}$ & $\begin{array}{l}\text { Mean: } 6 \text { per } \\
\text { month (range: } \\
1 \text { to } 17 \text { ) }\end{array}$ & \\
\hline & & & ASM only & $\begin{array}{l}25 \text { (range: } 12- \\
48)\end{array}$ & 24 & $\begin{array}{l}4 \text { years } \\
\text { (range: 5-71 } \\
\text { years) }\end{array}$ & & & & $\begin{array}{l}\text { CPS: } 7 \text { (29\%) } \\
\text { CPS + SG: } 10(42 \%)\end{array}$ & $\begin{array}{l}\text { Mean: } 12 \text { per } \\
\text { month (range: } \\
1 \text { to } 30 \text { ) }\end{array}$ & \\
\hline
\end{tabular}




\begin{tabular}{|c|c|c|c|c|c|c|c|c|c|c|c|c|}
\hline $\begin{array}{l}\text { 2Aluthor / } \\
\text { 2Publication } \\
22 \\
23\end{array}$ & Location & $\begin{array}{l}\text { Treatment } \\
\text { length }\end{array}$ & Comparators & $\begin{array}{l}\text { Duration of } \\
\text { Follow-up }\end{array}$ & $\begin{array}{l}\text { Sample } \\
\text { size, } n\end{array}$ & $\begin{array}{l}\text { Age at implant } \\
\text { mean (SD } \\
\text { [range]) }\end{array}$ & $\begin{array}{l}\text { Sex, F } \\
\text { (n [\%]) }\end{array}$ & $\begin{array}{l}\text { Mean } \\
\text { duration } \\
\text { of } \\
\text { disease }\end{array}$ & $\begin{array}{l}\text { Mean no. } \\
\text { of ASMs } \\
\text { used }\end{array}$ & Seizure types,,$^{\dagger} n\left(\%^{\ddagger}\right)$ & $\begin{array}{l}\text { Seizure } \\
\text { frequency }\end{array}$ & $\begin{array}{l}\text { Outcomes } \\
\text { Assessed }\end{array}$ \\
\hline $\begin{array}{l}24 \\
25 \\
26 \\
27 \\
27 \\
\end{array}$ & & & & & & & & & & $\begin{array}{l}\text { SPS/CPS + SG: } 1 \text { (4\%) } \\
\text { GTC: } 2(8 \%) \\
\text { SPS/CPS: } 2(8 \%) \\
\text { CPS + SG/psych: } 2(8 \%) \\
\end{array}$ & & \\
\hline \multirow{2}{*}{$\begin{array}{l}2 \text { Tgatum } 2001 \\
2 \text { [68] } \\
3 \text { (Gomparative } \\
3 \text { Prospective } \\
3 \text { case control) } \\
33\end{array}$} & \multirow[t]{2}{*}{ US } & \multirow[t]{2}{*}{$\begin{array}{l}13.2 \\
\text { months }\end{array}$} & VNS & \multirow[t]{2}{*}{$\begin{array}{l}13.2 \text { months } \\
\text { (NR) }\end{array}$} & 21 & $\begin{array}{l}24.8 \text { (range: } \\
4-51 \text { ) }\end{array}$ & $9(43)$ & $\begin{array}{l}17.0 \\
\text { (range: } \\
4-45 \text { ) }\end{array}$ & $\begin{array}{l}2.81 \\
\text { (range: 1- } \\
\text { 5) }\end{array}$ & \multirow[t]{2}{*}{ NR } & \multirow[t]{2}{*}{ NR } & \multirow{2}{*}{$\begin{array}{l}\text { Seizure } \\
\text { frequency, ASM } \\
\text { dose and usage, } \\
\text { QoL }\end{array}$} \\
\hline & & & $\begin{array}{l}\text { Control } \\
\text { (ASM) }\end{array}$ & & 21 & $26.1(5-57)$ & $12(57)$ & $\begin{array}{l}19.9 \\
\text { (range: } \\
3-46)\end{array}$ & $\begin{array}{l}2.38 \\
\text { (range: } 1- \\
\text { 4) }\end{array}$ & & & \\
\hline \multirow{2}{*}{$\begin{array}{l}33 \\
3 \text { Harden } 2000 \\
3[67] \\
\text { 3 (6omparative } \\
3 \text { Prospective } \\
3 \text { cohort) } \\
38 \\
30\end{array}$} & \multirow[t]{2}{*}{ US } & \multirow[t]{2}{*}{3 months } & VNS & $\begin{array}{l}3 \text { months (SD } \\
1.7)\end{array}$ & 20 & $\begin{array}{l}39 \text { (SD: } 9.1 \\
\text { [range: } 20- \\
\text { 58]) }\end{array}$ & $14(70)$ & \multirow[t]{2}{*}{ NR } & \multirow[t]{2}{*}{ NR } & $\begin{array}{l}\text { CPS: } 12(60 \%) \text { CP + } \\
\text { secondarily GTC: } 5(25 \%) \\
\text { Primary GTC: } 3(15 \%)\end{array}$ & $\begin{array}{l}\text { Mean: } 16.2 \\
\text { (SD 19.4) per } \\
\text { month }\end{array}$ & \multirow{2}{*}{$\begin{array}{l}\text { Seizure } \\
\text { frequency, } \\
\text { Medication side } \\
\text { effects, HRQoL }\end{array}$} \\
\hline & & & BMP (ASM) & $\begin{array}{l}.8 \text { months } \\
\text { (SD 1.6) }\end{array}$ & 20 & $\begin{array}{l}40.2 \text { (SD: } 13.3 \\
\text { [range: } 24- \\
69] \text { ]) }\end{array}$ & $14(70)$ & & & $\begin{array}{l}\text { CPS: } 10 \text { (50\%) Secondarily } \\
\text { GTC: } 5 \text { (25\%) CP + } \\
\text { Secondarily GTC: } 5 \text { (25\%) }\end{array}$ & $\begin{array}{l}\text { Mean: } 3.2 \text { (SD } \\
\text { 7.4) per } \\
\text { month }\end{array}$ & \\
\hline
\end{tabular}

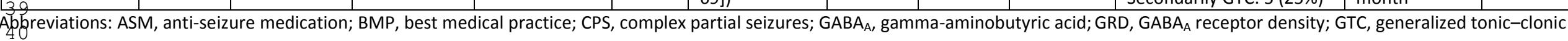

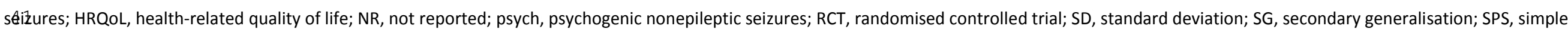
parztial seizures; SZ, seizure; VNS, Vagus Nerve Stimulation.

†4May be counted in more than one type; ¥ Where applicable; § Cyberonics-sponsored VNS study (Cyberonics is owned by LivaNova). 
Table 3: Summary of high and low stimulation parameters

\begin{tabular}{|l|l|l|l|l|l|l|}
\hline VNS Therapy Parameter & \multicolumn{2}{|l|}{ Landy 1993 } & \multicolumn{2}{l|}{ E-03 (Salinsky 1995) } & E-05 (Handforth 1998) \\
\hline Device setting & Low & High & Low & High & Low & High \\
\hline Output current $(\mathrm{mA})$ & $0.5-3.0$ & $0.5-3.0$ & $0.25-2.75$ & $0.25-3.0$ & 1.2 (avg) & 1.3 (avg) \\
\hline Signal frequency $(\mathrm{Hz})$ & $1-2$ & $20-50$ & $1-2$ & $20-50$ & 1 & 30 \\
\hline Pulse width $(\mu \mathrm{sec})$ & 130 & 500 & 130 & 500 & 130 & 500 \\
\hline Signal on time $(\mathrm{sec})$ & 30 & $30-90$ & 30 & $30-90$ & 30 & 30 \\
\hline Signal off time $(\mathrm{min})$ & $60-180$ & $5-10$ & $60-180$ & $5-10$ & 180 & 5 \\
\hline
\end{tabular}

\subsection{Participants experiencing $\geq 50 \%$ reduction in seizure frequency}

A total of six studies (three RCTs and three comparative observational studies) were included in the analysis. Overall, the pooled odds ratio (based on the results of RCTs and comparative observational studies) for experiencing $\geq 50 \%$ reduction in seizure frequency was statistically significantly greater in adult participants undergoing VNS Therapy compared with low stim VNS Therapy/BMP/ASM (OR: 2.27 [95\% Cl: 1.47, 3.51]; $p=0.0002)$. A similar statistically significant result was observed when results were pooled by study type (RCTs: OR 19.3 [95\% Cl: 1.16, 3.20], p=0.01; observational comparative studies: OR 3.64 [95\% Cl: 1.51, 8.73], p=0.004). Low levels of heterogeneity were observed between studies (Fig. 2).

\subsection{Participants experiencing $\geq 75 \%$ reduction in seizure frequency}

Five studies (two RCTs and three comparative observational studies) were included in the analysis. In the pooled analysis, the odds of experiencing a $\geq 75 \%$ reduction in seizure frequency were more than three times greater in adult participants undergoing VNS Therapy compared with low-stimulation VNS Therapy/ASM (OR: 3.56 [95\% Cl: 1.59, 7.98]; p=0.002). A similar statistically significant result was observed for pooled RCT studies (OR 5.54 [95\% Cl: 1.56, 19.67]; $p=0.008$ ); pooled results for comparative observational studies were not statistically significant (OR: 2.43 [95\% Cl: 0.83, 7.11]; $\mathrm{p}=0.11$ ). A trend for a greater VNS Therapy treatment effect in RCTs at a shorter follow-up time (OR 5.54 [95\% Cl: 1.56, 19.67]) compared with observational data at a longer follow-up time (OR: 2.43 [95\% Cl: $0.83,7.11]$ ) was observed. Low levels of heterogeneity were observed between studies (Fig. 3).

\subsection{Participants that are seizure free}

A total of six studies (two RCTs and four comparative observational studies) were included in the analysis. There is no difference in the odds of freedom from seizures in adult participants undergoing VNS Therapy compared with low-stimulation VNS Therapy/ASM (OR: 0.82 [95\% Cl: 0.37, 1.84]; p=0.64). On a study level, results were inconsistent across RCTs and comparative observational studies. 
Moderate levels of heterogeneity were observed between studies and there were large levels of uncertainty across the trial estimates due to low event numbers (Fig. 4).

\subsection{Mean change from baseline in seizure frequency}

Three RCT studies were included in the analysis. VNS Therapy was associated with a statistically significant decrease in the percentage change from baseline in seizures compared with low VNS Therapy (CFB: $-18.26 \%$ [95\% Cl: $-20.12,-16.41] ; p<0.00001)$. Consistent results were observed across the three RCTs reporting on this outcome and low levels of heterogeneity were observed (Fig. 5).

\subsection{ASM load}

The analysis for ASM load was based on two studies (one RCTs and one comparative observational studies). In the pooled analysis, participants undergoing VNS Therapy had a significant reduction in the risk of having an increased ASM load when compared with BMP or control (case-matched participants on ASMs) (risk ratio [RR]: 0.36 [95\% Cl: $0.21,0.62] ; p=0.0002$ ). Similarly, pooled analysis indicated that participants undergoing VNS Therapy had a significant reduction in the risk of adding one or more new ASMs during treatment when compared with BMP or control (case-matched participants on ASMs) (RR: 0.28 [95\% Cl: $0.13,0.58] ; p=0.0007)$. Results from a single RCT and comparative observational study formed the pooled analysis for both outcomes; low levels of heterogeneity were observed between studies. Separately, both studies reported significant differences for both outcomes favouring VNS Therapy (see Fig. 6 and Fig. 7).

\subsection{VNS Therapy discontinuation}

The discontinuation analysis included two RCT studies; no difference in the odds of discontinuing VNS Therapy treatment in adult participants undergoing VNS Therapy versus low-stimulation VNS Therapy/BMP was observed (OR: 1.31 [95\% Cl: 0.51, 3.36]; $p=0.57$ ). Consistent results were observed across the two RCTs reporting on this outcome. Low levels of heterogeneity were observed between studies and there were large levels of uncertainty across the trial estimates due to low event numbers (Fig. 8).

\subsection{SAES}

A single RCT study was included in the SAE analysis. No difference in the odds of an SAE in adult participants undergoing VNS Therapy compared with BMP was observed (OR: 1.87 [95\% Cl: 0.42, 8.24]; $\mathrm{p}=0.41$ ) (Fig. 9). 


\section{DISCUSSION}

This systematic review and meta-analysis demonstrated that in people with DRE, adjunctive highstimulation VNS Therapy resulted in statistically significant reductions in seizure frequency without increasing the rate of SAEs or discontinuations when compared with adjunctive low-stimulation VNS Therapy/ASM/best medical practice. This evidence validates the consideration of VNS Therapy for people who respond poorly to ASMs, or those who are unsuitable for or unwilling to undergo any cranial procedure. Furthermore, the results of this study are in agreement with the current guideline recommendations for the use of VNS Therapy in adults [27, 70-72].

While VNS Therapy resulted in a statistically significant outcomes at the pooled level, some were not statistically significant at the trial level. For the $\geq 50 \%$ reduction in seizure frequency outcome, only a single trial was statistically significant at the trial level (E03). The other studies (E-05 and PuLsE) were not statistically significant likely due to the low number of participants involved and wide confidence intervals observed. For the $\geq 75 \%$ reduction in seizure frequency outcome, the pooled analysis (RCTs and comparative observational studies) and pooled RCT analysis both reported a statistically significant benefit of VNS Therapy. However, the pooled results for the comparative observational studies were not statistically significant, possibly due to study heterogeneity (specifically participant number, study length) and different magnitudes of treatment effects.

There is no difference in the odds of complete freedom from seizures for adult participants undergoing VNS Therapy versus low-stimulation VNS Therapy/ASM. This result reflects current evidence in the literature, with other studies reporting that people with DRE undergoing VNS Therapy have a low rate of seizure freedom, despite response and seizure freedom rates increasing over time [73]. It must be noted that no events for seizure freedom were observed in RCT studies included in this analysis, with seizure freedom events only recorded in the comparative observational studies, which have a longer follow-up. Seizure freedom, however, was observed in 15 of 273 individuals with DRE.

The beneficial impact of VNS Therapy on ASM load was limited to two studies (PuLsE RCT and Tatum 2001), indicating that participants are less likely to require new ASMs or have an increased ASM load compared with BMP or control (case-matched participants on ASMs). When viewed alongside other seizure control outcomes from this analysis, the evidence suggests that VNS Therapy may permit the reduction in concomitant ASMs without loss of seizure control. A lower drug burden is clinically important because excessive drug load may be associated with decreased tolerability, and may 
consequently reduce the likelihood of seizure freedom [74]. Furthermore, certain ASMs are linked with a range of metabolic consequences that can adversely affect bone, lipid, and gonadal steroid metabolism. Consequently, reducing the drug burden may lower the risk of such complications [75]. Reductions in ASM load may also improve participant QoL, as a greater number of ASMs is a significant predictor of poor QoL [76]. Additionally, studies have shown that seizure frequency in people with DRE was one of the most important factors contributing to patient QoL $[77,78]$. Consequently, a reduction in seizures and their frequency may translate into QoL benefits. Of note, several studies which investigated use of VNS Therapy in individuals with DRE report improvements in seizure control and also observed improvements in QoL $[35,67,79]$.

VNS therapy has comparable safety outcomes, specifically for SAEs and discontinuations, when compared with low-stimulation VNS Therapy/best medical practice. There was no difference in the odds of discontinuing treatment in adult participants undergoing VNS Therapy versus low-stimulation VNS Therapy/best medical practice, and there was no difference in the odds of an SAE in adult participants undergoing VNS Therapy versus best medical practice. When viewed alongside the seizure control outcomes from this analysis, the safety evidence suggests that VNS Therapy may facilitate better seizure control without increasing the rate of discontinuation or SAEs compared with participants undergoing VNS Therapy versus low-stimulation VNS Therapy/best medical practice. The discontinuation analysis was based on two RCTs of different duration; 12 months (PuLSE) and 3.4-4.5 months (E-05). Of note, there was only a single event in the VNS Therapy and comparator arm for E-05 compared with 46 and 47 events in the VNS Therapy and comparator arm for PuLsE. The main reasons for discontinuation in E-05 were Cheyne-Stokes respiration $(n=1)$, and a variety of unspecified symptoms $(n=1)$. For PuLsE, the majority of study discontinuations in either treatment group were due to premature termination of the study by the sponsor, and there were no discontinuations due to AEs.

It must be noted that of the studies identified for the meta-analysis, there was only one RCT (PuLsE; which had its outcomes restricted to 12 months for the meta-analysis) [35] and two comparative observational studies $[63,64]$ which reported long-term outcomes ( $\geq 2$ years). Consequently, this makes it difficult to determine the long-term benefits associated with VNS Therapy. However, there are noncomparative, single-arm studies of VNS Therapy in people with DRE which provide an insight into the long-term treatment effects of VNS Therapy. A retrospective analysis of 436 participants (predominantly adults) with DRE treated with VNS Therapy reported that participants achieved a mean seizure reduction of $55.8 \%$ after a mean follow-up of 5 years; 40.5 and $63.75 \%$ of participants achieved $\geq 75 \%$ 
seizure control and $\geq 50 \%$ seizure control, respectively [80]. The mean reduction in seizures continued to improve with duration; of those participants with $>10$ years of follow-up $(n=65)$, the mean decrease in seizure frequency at last follow-up was 76.3\% [81]. Additionally, results from a prospective, open-label study of long-term VNS Therapy use (2 years) in individuals with DRE $(n=40)$ reported no significant safety events associated with Therapy and 95\% (38/40) of patients remained on VNS Therapy for the study duration (one patient died [SUDEP] and the other was lost to follow-up after 1 year of treatment) [82]. The long-term benefits of VNS Therapy are reported in a number of other single-arm studies [8385]. These results highlight the long-term benefits of VNS Therapy for people with DRE, but long-term comparative studies are required to determine if the benefits observed were solely due to VNS Therapy, or a potentially synergistic combination of ASM regimens and VNS Therapy. There are single-arm studies of shorter duration which support the meta-analysis results for VNS Therapy and ASM load. DeGiorgio et al, 2000 reported that participants with refractory epilepsy $(n=195)$ who were treated with VNS Therapy had a reduction in the mean number of ASMs, from 2.3 to 2.1 at the end of 12 months [46]. In addition, another study reported that up to $40 \%$ of participants experienced a decrease in the total dose of ASMs after 12 months of VNS Therapy [86]. While positive, these observations need to be supported by longterm comparative studies.

As with all systematic reviews and meta-analyses, the results may need to be interpreted with caution due to certain limitations which include inconsistency across the trials for length of follow-up, greater treatment effects were often observed with observational comparative studies versus RCTs, and there were a very limited number of studies $(\leq 2)$ for certain meta-analysis outcomes, specifically the discontinuation, SAE, and ASM load analyses. Of note, the number of studies identified for the metaanalysis was limited as the analysis focused on comparative observational studies and RCTs (which are the gold standard for generating estimates of relative treatment effects) which can be viewed as a strength of this analysis. Overall, there is limited high-quality evidence supporting the use of VNS Therapy in DRE. In addition, many trial-level estimates are associated with large levels of uncertainty (wide Cls) due to low participant and event numbers and in some instances single events are driving the direction of treatment effects. There was substantial variation in baseline seizure frequency reported by observational comparative studies (0.1-3.5 seizures per day). Seizure frequency in VNS Therapy participants and control participants were not comparable at baseline in the majority of reporting studies, with participants in the VNS Therapy arm having a greater baseline seizure frequency [63, 66, 67]. Another limitation of the analysis was the differences in VNS Therapy stimulation parameters across studies contributing to further heterogeneity amongst participant groups. In the early RCTs regulating 
stimulation parameters, the low-stimulation group was titrated to sensation and the high stimulation group to maximum tolerated stimulation. Subsequent studies have suggested this may not be necessary for optimal efficacy and may contribute to difficulties in tolerability. Lastly, three of the VNS Therapy trials informing efficacy (E-03, E-05 and Landy 1993) did not compare VNS Therapy with ASM therapy only. These trials compared VNS Therapy at 'high stimulation' settings with a presumed sub-therapeutic 'low-stimulation' regimen; ASMs were given in both arms. Therapeutic VNS is driven by the generation of action potentials along the vagus nerve, which is a function of the strength-duration relationship [87]. It is reported in the literature that $1.5 \mathrm{~mA}$ at $130 \mu \mathrm{sec}$ to $2.25 \mathrm{~mA}$ at $500 \mu \mathrm{sec}$ is considered a therapeutic dose $[20,62,69,88]$. Based on the reported data, the low-stimulation arms in each of these trials contain patients that could fall within this therapeutic range (see Table 3). Consequently, any residual benefit of 'low-stimulation' may have resulted in the overestimation of the efficacy of ASMs in the lowstimulation group.

This study has highlighted areas of focus for future research. There is a need for comparative studies assessing the long-term efficacy and safety of VNS Therapy as an adjunct to ASMs compared with relevant comparators. In addition, more research is required to reinforce the positive results observed for ASM load when VNS Therapy is used as an adjunct to ASMs

\section{Conclusions}

Although there is much literature devoted to VNS Therapy, there is a paucity of comparative data and this should be a focus for future research. This meta-analysis demonstrated the benefits of VNS Therapy in people with DRE, which included improvement in seizure frequency without an increase in the rate of SAEs or discontinuations. The evidence validates the consideration of VNS Therapy for people who are not responding to ASMs, or those who are unsuitable for or unwilling to undergo cranial procedures. 


\section{List of figures:}

Fig. 1 PRISMA flow diagram

Abbreviations: RCT, randomised controlled trial; VNS, Vagus Nerve Stimulation.

* Primarily due to publications including non-relevant comparators, no outcomes of interest or publications were superseded by a linked publication

Fig. 2 Participants experiencing $\geq 50 \%$ reduction in seizure frequency Abbreviations: ASM, antiepileptic drug; $\mathrm{BMP}$, best medical practice; $\mathrm{Cl}$, confidence interval; $\mathrm{RCT}$, randomised controlled trial; SD, standard deviations; VNS, Vagus Nerve Stimulation.

Fig. 3 Participants experiencing $\geq 75 \%$ reduction in seizure frequency Abbreviations: ASM, antiepileptic drug; $\mathrm{Cl}$, confidence interval; $\mathrm{RCT}$, randomised controlled trial; SD, standard deviations; VNS, Vagus Nerve Stimulation.

Fig. 4 Seizure-free participants Abbreviations: ASM, antiepileptic drug; $\mathrm{Cl}$, confidence interval; $\mathrm{RCT}$, randomised controlled trial; SD, standard deviation; VNS, Vagus Nerve Stimulation.

Fig. 5 Change from baseline in seizures, percentage Abbreviations: ASM, antiepileptic drug; $\mathrm{Cl}$, confidence interval; $\mathrm{RCT}$, randomised controlled trial; SD, standard deviation; VNS, Vagus Nerve Stimulation.

Fig. 6 Number of participants with increased ASM load Abbreviations: ASM, anti-seizure medication; $\mathrm{Cl}$, confidence interval; $\mathrm{RCT}$, randomised controlled trial; VNS, Vagus Nerve Stimulation.

Fig. 7 Number of participants with one or more new ASMs Abbreviations: ASM, anti-seizure medication; $\mathrm{Cl}$, confidence interval; $\mathrm{RCT}$, randomised controlled trial; VNS, Vagus Nerve Stimulation.

Fig. 8 Treatment discontinuations Abbreviations: ASM, anti-seizure medication; BMP, best medical practice; $\mathrm{Cl}$, confidence interval; RCT, randomised controlled trial; VNS, Vagus Nerve Stimulation. 


\section{Fig. 9 SAES}

Abbreviations: ASM, anti-seizure medication; BMP, best medical practice; $\mathrm{Cl}$, confidence interval; $\mathrm{RCT}$, randomised controlled trial; SAE, serious adverse event; VNS, Vagus Nerve Stimulation. 


\section{References}

1. World Health Organization (WHO) (2019) Epilepsy Overview. Available from: https://www.who.int/health-topics/epilepsy. Accessed on: 16th July 2021

2. Kalilani L, Sun X, Pelgrims B, Noack-Rink M, Villanueva V (2018) The epidemiology of drugresistant epilepsy: A systematic review and meta-analysis. Epilepsia 59(12):2179-93 https://doi.org/10.1111/epi.14596.

3. Kwan P, Arzimanoglou A, Berg AT, Brodie MJ, Allen Hauser W, Mathern G, et al. (2010) Definition of drug resistant epilepsy: consensus proposal by the ad hoc Task Force of the ILAE Commission on Therapeutic Strategies. Epilepsia 51(6):1069-77 https://doi.org/10.1111/j.15281167.2009.02397.x.

4. Strzelczyk A, Griebel C, Lux W, Rosenow F, Reese J-P (2017) The Burden of Severely DrugRefractory Epilepsy: A Comparative Longitudinal Evaluation of Mortality, Morbidity, Resource Use, and Cost Using German Health Insurance Data. Frontiers in neurology 8(712https://doi.org/10.3389/fneur.2017.00712.

5. Kantanen AM, Reinikainen M, Parviainen I, Kälviäinen R (2017) Long-term outcome of refractory status epilepticus in adults: A retrospective population-based study. Epilepsy research 133(13-21 https://doi.org/10.1016/j.eplepsyres.2017.03.009.

6. Wang T, Wang J, Dou Y, Yan W, Ding D, Lu G, et al. (2020) Clinical characteristics and prognosis in a large paediatric cohort with status epilepticus. Seizure 80(5-11 https://doi.org/10.1016/j.seizure.2020.04.001.

7. Tian L, Li Y, Xue X, Wu M, Liu F, Hao X, et al. (2015) Super-refractory status epilepticus in West China. Acta neurologica Scandinavica 132(1):1-6 https://doi.org/10.1111/ane.12336.

8. Novak JL, Miller PR, Markovic D, Meymandi SK, DeGiorgio CM (2015) Risk Assessment for Sudden Death in Epilepsy: The SUDEP-7 Inventory. Frontiers in neurology 6(252) https://doi.org/10.3389/fneur.2015.00252.

9. Ryvlin P, Cucherat M, Rheims S (2011) Risk of sudden unexpected death in epilepsy in patients given adjunctive antiepileptic treatment for refractory seizures: a meta-analysis of placebocontrolled randomised trials. The Lancet Neurology 10(11):961-8 https://doi.org/10.1016/s1474-4422(11)70193-4.

10. Devinsky O, Hesdorffer DC, Thurman DJ, Lhatoo S, Richerson G (2016) Sudden unexpected death in epilepsy: epidemiology, mechanisms, and prevention. The Lancet Neurology 15(10):1075-88 https://doi.org/10.1016/s1474-4422(16)30158-2.

11. Ridsdale L, Wojewodka G, Robinson E, Landau S, Noble A, Taylor S, et al. (2017) Characteristics associated with quality of life among people with drug-resistant epilepsy. Journal of neurology 264(6):1174-84 https://doi.org/10.1007/s00415-017-8512-1.

12. Brodie MJ (2013) Road to refractory epilepsy: the Glasgow story. Epilepsia 54 Suppl 2(5-8 https://doi.org/10.1111/epi.12175.

13. Chen Z, Brodie MJ, Liew D, Kwan P (2018) Treatment Outcomes in Patients With Newly Diagnosed Epilepsy Treated With Established and New Antiepileptic Drugs: A 30-Year Longitudinal Cohort Study. JAMA neurology 75(3):279-86 https://doi.org/10.1001/jamaneurol.2017.3949.

14. Epilepsy Action (2020) Epilepsy brain surgery for adults. Available from: https://www.epilepsy.org.uk/info/treatment/surgery/adult\#brain1 Accessed on: 16th July 2021

15. Masino SA, Rho JM (2012) Mechanisms of Ketogenic Diet Action. In: Noebels JL, Avoli M, Rogawski MA, al. e, editors. Jasper's Basic Mechanisms of the Epilepsies, 4th edition. Bethesda (MD): National Center for Biotechnology Information (US). 
16. Zangiabadi N, Ladino LD, Sina F, Orozco-Hernández JP, Carter A, Téllez-Zenteno JF (2019) Deep Brain Stimulation and Drug-Resistant Epilepsy: A Review of the Literature. Frontiers in neurology 10(601- https://doi.org/10.3389/fneur.2019.00601.

17. Epilepsy Foundation (2017) Responsive Neurostimulation (RNS). Available from: https://www.epilepsy.com/learn/treating-seizures-and-epilepsy/devices/responsiveneurostimulation-rns. Accessed on: 16th July 2021

18. Ben-Menachem E, Revesz D, Simon BJ, Silberstein S (2015) Surgically implanted and non-invasive vagus nerve stimulation: a review of efficacy, safety and tolerability. European journal of neurology 22(9):1260-8 https://doi.org/10.1111/ene.12629.

19. PR Newswire (2012) NeuroSigma Receives CE Certification. Available from: https://www.prnewswire.com/news-releases/neurosigma-receives-ce-certification168578146.html. Accessed on: 21 July 2021

20. Handforth A, DeGiorgio CM, Schachter SC, Uthman BM, Naritoku DK, Tecoma ES, et al. (1998) Vagus nerve stimulation therapy for partial-onset seizures: a randomized active-control trial. Neurology 51(1):48-55 https://doi.org/10.1212/wnl.51.1.48.

21. The Vagus Nerve Stimulation Study Group (1995) A randomized controlled trial of chronic vagus nerve stimulation for treatment of medically intractable seizures. Neurology 45(2):224-30 https://doi.org/10.1212/wnl.45.2.224.

22. Sheng J, Liu S, Qin H, Li B, Zhang X (2018) Drug-Resistant Epilepsy and Surgery. Current neuropharmacology 16(1):17-28 https://doi.org/10.2174/1570159x15666170504123316.

23. de Tisi J, Bell GS, Peacock JL, McEvoy AW, Harkness WF, Sander JW, et al. (2011) The long-term outcome of adult epilepsy surgery, patterns of seizure remission, and relapse: a cohort study. Lancet (London, England) 378(9800):1388-95 https://doi.org/10.1016/s0140-6736(11)60890-8.

24. Ryvlin P, Rheims S (2008) Epilepsy surgery: eligibility criteria and presurgical evaluation. Dialogues Clin Neurosci 10(1):91-103.

25. Liu G, Slater N, Perkins A (2017) Epilepsy: Treatment Options. American family physician 96(2):87-96.

26. Englot DJ, Birk H, Chang EF (2017) Seizure outcomes in nonresective epilepsy surgery: an update. Neurosurgical review 40(2):181-94 https://doi.org/10.1007/s10143-016-0725-8.

27. NHS Commissioning Board (2013) Clinical Commissioning Policy: Vagal Nerve Stimulation for Epilepsy. 01 August 2021).

28. LivaNova VNS Therapy ${ }^{\circledR}$ System Epilepsy Physician's Manual. August 2020. OUS version.

29. LivaNova VNS Therapy ${ }^{\circledR}$ System Epilepsy Physician’s Manual. May 2020. US version.

30. LivaNova Data on file (Certification Notice CE Marking [KCS/MD - 37600] May 1, 1994.

31. Food and Drug Administration (FDA) (1997) Premarket Approval (PMA) P970003. VNS THERAPY SYSTEM. Available from: https://www.accessdata.fda.gov/scripts/cdrh/cfdocs/cfpma/pma.cfm?id=P970003 Accessed on: 21 July 2021

32. Page MJ, McKenzie JE, Bossuyt PM, Boutron I, Hoffmann TC, Mulrow CD, et al. (2021) The PRISMA 2020 statement: An updated guideline for reporting systematic reviews. Journal of clinical epidemiology 134(178-89 https://doi.org/10.1016/j.jclinepi.2021.03.001.

33. National Institute for Health and Care Excellence (NICE) (2015) Single technology appraisal: User guide for company evidence submission template (PMG24). Available from: https://www.nice.org.uk/process/pmg24/resources/single-technology-appraisal-user-guide-forcompany-evidence-submission-template-pdf-72286715419333 Accessed on: 01 July 2021

34. Effective Public Health Practice Project (EPHPP) (2021) Quality Assessment Tool for Quantitative Studies. Available from: https://www.ephpp.ca/quality-assessment-tool-for-quantitativestudies/. Accessed on: 01 July 2021 
35. Ryvlin P, Gilliam FG, Nguyen DK, Colicchio G, ludice A, Tinuper P, et al. (2014) The long-term effect of vagus nerve stimulation on quality of life in patients with pharmacoresistant focal epilepsy: the PuLsE (Open Prospective Randomized Long-term Effectiveness) trial. Epilepsia 55(6):893-900 https://doi.org/10.1111/epi.12611.

36. Ben-Menachem E, Mañon-Espaillat R, Ristanovic R, Wilder BJ, Stefan H, Mirza W, et al. (1994) Vagus nerve stimulation for treatment of partial seizures: 1 . A controlled study of effect on seizures. First International Vagus Nerve Stimulation Study Group. Epilepsia 35(3):616-26 https://doi.org/10.1111/j.1528-1157.1994.tb02482.x.

37. Ramsay RE, Uthman BM, Augustinsson LE, Upton AR, Naritoku D, Willis J, et al. (1994) Vagus nerve stimulation for treatment of partial seizures: 2 . Safety, side effects, and tolerability. First International Vagus Nerve Stimulation Study Group. Epilepsia 35(3):627-36 https://doi.org/10.1111/j.1528-1157.1994.tb02483.x.

38. Holder LK, Wernicke JF, Tarver WB (1992) Treatment of refractory partial seizures: preliminary results of a controlled study. Pacing and clinical electrophysiology : PACE 15(10 Pt 2):1557-71 https://doi.org/10.1111/j.1540-8159.1992.tb02934.x.

39. Morris GL, 3rd (2003) A retrospective analysis of the effects of magnet-activated stimulation in conjunction with vagus nerve stimulation therapy. Epilepsy \& behavior : E\&B 4(6):740-5 https://doi.org/10.1016/j.yebeh.2003.08.025.

40. Michael JE, Wegener K, Barnes DW (1993) Vagus nerve stimulation for intractable seizures: one year follow-up. The Journal of neuroscience nursing : journal of the American Association of Neuroscience Nurses 25(6):362-6 https://doi.org/10.1097/01376517-199312000-00007.

41. George R, Salinsky M, Kuzniecky R, Rosenfeld W, Bergen D, Tarver WB, et al. (1994) Vagus nerve stimulation for treatment of partial seizures: 3 . Long-term follow-up on first 67 patients exiting a controlled study. First International Vagus Nerve Stimulation Study Group. Epilepsia 35(3):63743 https://doi.org/10.1111/j.1528-1157.1994.tb02484.x.

42. Holder LK, Wernicke J, Tarver WB (1993) Long-term follow-up of 37 patients with refractory partial seizures treated with vagus nerve stimulation. Journal of Epilepsy 6(4):206-14.

43. Amar AP, Heck CN, Levy ML, Smith T, DeGiorgio CM, Oviedo S, et al. (1998) An institutional experience with cervical vagus nerve trunk stimulation for medically refractory epilepsy: rationale, technique, and outcome. Neurosurgery 43(6):1265-76; discussion 76-80 https://doi.org/10.1097/00006123-199812000-00001.

44. Dodrill CB, Morris GL (2001) Effects of Vagal Nerve Stimulation on Cognition and Quality of Life in Epilepsy. Epilepsy \& behavior : E\&B 2(1):46-53 https://doi.org/10.1006/ebeh.2000.0148.

45. Amar AP, DeGiorgio CM, Tarver WB, Apuzzo ML (1999) Long-term multicenter experience with vagus nerve stimulation for intractable partial seizures: results of the XE5 trial. Stereotactic and functional neurosurgery 73(1-4):104-8 https://doi.org/10.1159/000029764.

46. DeGiorgio CM, Schachter SC, Handforth A, Salinsky M, Thompson J, Uthman B, et al. (2000) Prospective long-term study of vagus nerve stimulation for the treatment of refractory seizures. Epilepsia 41(9):1195-200 https://doi.org/10.1111/j.1528-1157.2000.tb00325.x.

47. DeGiorgio CM, Thompson J, Lewis P, Arrambide S, Naritoku D, Handforth A, et al. (2001) Vagus nerve stimulation: analysis of device parameters in 154 patients during the long-term XE5 study. Epilepsia 42(8):1017-20 https://doi.org/10.1046/j.1528-1157.2001.0420081017.x.

48. Bunch S, DeGiorgio CM, Krahl S, Britton J, Green P, Lancman M, et al. (2007) Vagus nerve stimulation for epilepsy: is output current correlated with acute response? Acta neurologica Scandinavica 116(4):217-20 https://doi.org/10.1111/j.1600-0404.2007.00878.x.

49. DeGiorgio C, Heck C, Bunch S, Britton J, Green P, Lancman M, et al. (2005) Vagus nerve stimulation for epilepsy: randomized comparison of three stimulation paradigms. Neurology 65(2):317-9 https://doi.org/10.1212/01.wnl.0000168899.11598.00. 
50. Wang AJ, Bick SK, Williams ZM (2020) Vagus Nerve Stimulation versus Responsive Neurostimulator System in Patients with Temporal Lobe Epilepsy. Stereotactic and functional neurosurgery 98(1):21-9 https://doi.org/10.1159/000504859.

51. Bhraguvansh A (2020) VNS and RNS patients are driving: The impact of seizure freedom and reduction American Epilepsy Society. Abstract 154.

52. Ellens NR, Elisevich K, Burdette DE, Patra SE (2018) A Comparison of Vagal Nerve Stimulation and Responsive Neurostimulation for the Treatment of Medically Refractory Complex Partial Epilepsy. Stereotactic and functional neurosurgery 96(4):259-63 https://doi.org/10.1159/000492232.

53. Kuba R, Novák Z, Chrastina J, Pažourková M, Hermanová M, Ošlejšková H, et al. (2013) Comparing the effects of cortical resection and vagus nerve stimulation in patients with nonlesional extratemporal epilepsy. Epilepsy \& behavior : E\&B 28(3):474-80 https://doi.org/10.1016/j.yebeh.2013.05.036.

54. Wheless JW, Isojarvi J, Lee D, Drummond R, Benbadis SR (2014) Clobazam is efficacious for patients across the spectrum of disease severity of Lennox-Gastaut syndrome: post hoc analyses of clinical trial results by baseline seizure-frequency quartiles and VNS experience. Epilepsy \& behavior : E\&B 41(47-52 https://doi.org/10.1016/j.yebeh.2014.09.019.

55. Nei M, O'Connor M, Liporace J, Sperling MR (2006) Refractory generalized seizures: response to corpus callosotomy and vagal nerve stimulation. Epilepsia 47(1):115-22 https://doi.org/10.1111/j.1528-1167.2006.00377.x.

56. McGlone J, Valdivia I, Penner M, Williams J, Sadler RM, Clarke DB (2008) Quality of life and memory after vagus nerve stimulator implantation for epilepsy. The Canadian journal of neurological sciences Le journal canadien des sciences neurologiques 35(3):287-96 https://doi.org/10.1017/s0317167100008854.

57. Lim Z, Wong K, Downs J, Bebbington K, Demarest S, Leonard H (2018) Vagus nerve stimulation for the treatment of refractory epilepsy in the CDKL5 Deficiency Disorder. Epilepsy research 146(36-40 https://doi.org/10.1016/j.eplepsyres.2018.07.013.

58. Ben-Menachem E, Hamberger A, Hedner T, Hammond EJ, Uthman BM, Slater J, et al. (1995) Effects of vagus nerve stimulation on amino acids and other metabolites in the CSF of patients with partial seizures. Epilepsy research 20(3):221-7 https://doi.org/10.1016/09201211(94)00083-9.

59. Drees CS, McDermott D, Abosch D, Ojemann A, Brown MG (2018) Responsive neurostimulation (RNS) use in patients with and without prior vagus nerve stimulator (VNS) implantation. American Epilepsy Society. Abst. 1.165.

60. Jamy R, Kaur M, Pizarro D, Toth E, Pati S (2019) Practice trends and the outcome of neuromodulation therapies in epilepsy: A single-center study. Epilepsia open 4(3):493-7 https://doi.org/10.1002/epi4.12345.

61. Boon P, Vandekerckhove T, Achten E, Thiery E, Goossens L, Vonck K, et al. (1999) Epilepsy surgery in Belgium, the experience in Gent. Acta neurologica Belgica 99(4):256-65.

62. Landy HJ, Ramsay RE, Slater J, Casiano RR, Morgan R (1993) Vagus nerve stimulation for complex partial seizures: surgical technique, safety, and efficacy. Journal of neurosurgery 78(1):26-31 https://doi.org/10.3171/jns.1993.78.1.0026.

63. Boon P, D'Havé M, Van Walleghem P, Michielsen G, Vonck K, Caemaert J, et al. (2002) Direct medical costs of refractory epilepsy incurred by three different treatment modalities: a prospective assessment. Epilepsia 43(1):96-102 https://doi.org/10.1046/j.15281157.2002.40100.x. 
64. Gonen OM, Gandelman-Marton R, Kipervasser S, Neufeld MY (2015) The prognosis of refractory epilepsy patients rejected from epilepsy surgery. Acta neurologica Scandinavica 131(1):58-62 https://doi.org/10.1111/ane.12311.

65. Marrosu F, Serra A, Maleci A, Puligheddu M, Biggio G, Piga M (2003) Correlation between $\mathrm{GABA}(\mathrm{A})$ receptor density and vagus nerve stimulation in individuals with drug-resistant partial epilepsy. Epilepsy research 55(1-2):59-70 https://doi.org/10.1016/s0920-1211(03)00107-4.

66. Hoppe C, Wagner L, Hoffmann JM, von Lehe M, Elger CE (2013) Comprehensive long-term outcome of best drug treatment with or without add-on vagus nerve stimulation for epilepsy: a retrospective matched pairs case-control study. Seizure 22(2):109-15 https://doi.org/10.1016/j.seizure.2012.11.003.

67. Harden CL, Pulver MC, Ravdin LD, Nikolov B, Halper JP, Labar DR (2000) A Pilot Study of Mood in Epilepsy Patients Treated with Vagus Nerve Stimulation. Epilepsy \& behavior : E\&B 1(2):93-9 https://doi.org/10.1006/ebeh.2000.0046.

68. Tatum WO, Johnson KD, Goff S, Ferreira JA, Vale FL (2001) Vagus nerve stimulation and drug reduction. Neurology 56(4):561-3 https://doi.org/10.1212/wnl.56.4.561.

69. Salinsky MC, Uthman BM, Ristanovic RK, Wernicke JF, Tarver WB (1996) Vagus nerve stimulation for the treatment of medically intractable seizures. Results of a 1-year open-extension trial. Vagus Nerve Stimulation Study Group. Archives of neurology 53(11):1176-80 https://doi.org/10.1001/archneur.1996.00550110128021.

70. Morris GL, Gloss D, Buchhalter J, Mack KJ, Nickels K, Harden C (2013) Evidence-based guideline update: vagus nerve stimulation for the treatment of epilepsy: report of the Guideline Development Subcommittee of the American Academy of Neurology. Neurology 81(16):1453-9.

71. Deutsche Gesellschaft für Neurologie (DGN) (2017) Erster epileptischer Anfall und Epilepsien im Erwachsenenalter. Available from: https://www.awmf.org/uploads/tx_szleitlinien/030041I_S1_Erster-epileptischer-Anfall_Epilespien_2018-05.pdf. Accessed on: 01 August 2021

72. Lega Italiana Contro I'Epilessia (LICE)/Società Italiana di Medicina Generale (SIMG) Guida pratica per la gestione del paziente affetto da epilessia. Available from: https://www.lice.it/LICE_ita/documenti_LICE-SIMG/pdf/1_clinica.pdf. Accessed on: 01 August 2021

73. Englot DJ, Chang EF, Auguste KI (2011) Vagus nerve stimulation for epilepsy: a meta-analysis of efficacy and predictors of response. Journal of neurosurgery 115(6):1248-55 https://doi.org/10.3171/2011.7.Jns11977.

74. Brodie MJ, Sills GJ (2011) Combining antiepileptic drugs--rational polytherapy? Seizure 20(5):369-75 https://doi.org/10.1016/j.seizure.2011.01.004.

75. Mintzer S (2010) Metabolic consequences of antiepileptic drugs. Current opinion in neurology 23(2):164-9 https://doi.org/10.1097/WCO.0b013e32833735e7.

76. George J, Kulkarni C, Sarma GRK (2015) Antiepileptic Drugs and Quality of Life in Patients with Epilepsy: A Tertiary Care Hospital-Based Study. Value in health regional issues 6(1-6 https://doi.org/10.1016/j.vhri.2014.07.009.

77. Guekht AB, Mitrokhina TV, Lebedeva AV, Dzugaeva FK, Milchakova LE, Lokshina OB, et al. (2007) Factors influencing on quality of life in people with epilepsy. Seizure 16(2):128-33 https://doi.org/10.1016/j.seizure.2006.10.011.

78. Kalitesi IDEHY (2019) Quality of life in patients with drug resistant epilepsy. Turk J Neurol 25(159-63.

79. Kawai K, Tanaka T, Baba H, Bunker M, Ikeda A, Inoue Y, et al. (2017) Outcome of vagus nerve stimulation for drug-resistant epilepsy: the first three years of a prospective Japanese registry. Epileptic disorders : international epilepsy journal with videotape 19(3):327-38 https://doi.org/10.1684/epd.2017.0929. 
80. Elliott RE, Morsi A, Kalhorn SP, Marcus J, Sellin J, Kang M, et al. (2011) Vagus nerve stimulation in 436 consecutive patients with treatment-resistant epilepsy: long-term outcomes and predictors of response. Epilepsy \& behavior : E\&B 20(1):57-63 https://doi.org/10.1016/j.yebeh.2010.10.017.

81. Elliott RE, Morsi A, Tanweer O, Grobelny B, Geller E, Carlson C, et al. (2011) Efficacy of vagus nerve stimulation over time: review of 65 consecutive patients with treatment-resistant epilepsy treated with VNS > 10 years. Epilepsy \& behavior : E\&B 20(3):478-83 https://doi.org/10.1016/j.yebeh.2010.12.042.

82. Huf RL, Mamelak A, Kneedy-Cayem K (2005) Vagus nerve stimulation therapy: 2-year prospective open-label study of 40 subjects with refractory epilepsy and low IQ who are living in long-term care facilities. Epilepsy \& behavior : E\&B 6(3):417-23 https://doi.org/10.1016/j.yebeh.2005.01.009.

83. Abubakr A, Wambacq I (2008) Long-term outcome of vagus nerve stimulation therapy in patients with refractory epilepsy. Journal of clinical neuroscience : official journal of the Neurosurgical Society of Australasia 15(2):127-9 https://doi.org/10.1016/j.jocn.2007.07.083.

84. Kuba R, Brázdil M, Kalina M, Procházka T, Hovorka J, Nezádal T, et al. (2009) Vagus nerve stimulation: longitudinal follow-up of patients treated for 5 years. Seizure 18(4):269-74 https://doi.org/10.1016/j.seizure.2008.10.012.

85. Nakken KO, Henriksen O, Røste GK, Lossius R (2003) Vagal nerve stimulation--the Norwegian experience. Seizure 12(1):37-41 https://doi.org/10.1016/s1059131102001383.

86. McLachlan RS, Sadler M, Pillay N, Guberman A, Jones M, Wiebe S, et al. (2003) Quality of life after vagus nerve stimulation for intractable epilepsy: is seizure control the only contributing factor? European neurology 50(1):16-9 https://doi.org/10.1159/000070853.

87. Geddes LA, Bourland JD (1985) The strength-duration curve. IEEE transactions on bio-medical engineering 32(6):458-9 https://doi.org/10.1109/tbme.1985.325456.

88. Fisher B, DesMarteau JA, Koontz EH, Wilks SJ, Melamed SE (2020) Responsive Vagus Nerve Stimulation for Drug Resistant Epilepsy: A Review of New Features and Practical Guidance for Advanced Practice Providers. Frontiers in neurology 11(610379 https://doi.org/10.3389/fneur.2020.610379. 
Records identified through database searching $(n=10,177)$

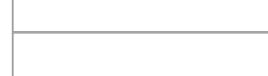

Duplicates removed

Records screened based on title and abstract
$(n=8,845)$

Records exclude Study design $(n=1,340)$
Disease $(n=2,609)$ Intervention ( $n=649)$ Population ( $n=67)$

Review/editorial $(n=462)$

Duplicate $(\mathrm{n}=1,886)$

Animal/in-vitro $(n=480)$

Protocol $(\mathrm{n}=373)$

Pre-2017 abstract $(n=216)$

Full-text articles assessed

Linked publication ( $\mathrm{n}=154$

$$
\begin{gathered}
\text { for eligibility } \\
(\mathrm{n}=607)
\end{gathered}
$$

$$
\text { Language }(\mathrm{n}=2)
$$

Additional records identifie

$(n=33)$

Full-text articles excluded, with

reasons $(n=425)$

Pooled analyses $(n=26)$
Study design $(n=2)$

Outcomes ( $\mathrm{n}=2$ )

Tagged relevant review $(n=90)$

Tagged non-comparative study
$(\mathrm{n}=305)$

Identified publications ( $\mathrm{n}=215)$

$\mathrm{RCTs,} \mathrm{n}=149$ on 101 unique studies

Non-RCTs, $n=66$ on 65 unique

Non-VNS-related publications

( $\mathrm{n}=167)$

Identified VNS publication

$\mathrm{RCTs}, \mathrm{n}=24$ on 7 unique studies

Non-RCTs, $n=24$ on 23 unique

$\checkmark$

Not eligible for inclusion in
meta-analysis* $(n=38)$

VNS publications for
meta-analysis $(n=10)$

RCTs, $n=4$ on 4 unique studies

Non-RCTs, $n=6$ on 6 unique studies 
Study or Subgroup

E03 (3.5 months) [RCT: High VNS vs low VNS]

E05 (3.4-4.5 months) [RCT: High VNS vs low VNS]

Subtotal $(95 \% \mathrm{Cl})$

Total events

Heterogeneity: $\mathrm{Chi}^{2}=0.03, \mathrm{df}=1(\mathrm{P}=0.87) ; \mathrm{I}^{2}=0 \%$

Test for overall effect. $Z=2.64(P=0.008)$

6.1.3 Comparative observational studies (adults)

Harden 2000 (3-3.8 months) [obs: VNS vs AEDs]

Hoppe 2013 ( 80.4 months) [obs: VNS vS AEDs]

Marrosu 2003 (1 (1 year) [obs: VNS vs control]

Subtotal $(95 \% \mathrm{Cl})$

Total events

Hetogenenty: $\mathrm{Chi}^{2}=1.31, \mathrm{df}=2(P=0.52) ; \mathrm{I}^{2}=0 \%$

\section{Total $(95 \% \mathrm{Cl})$}

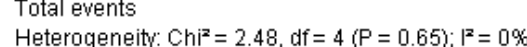

Test for overall effect: $Z=3.08(P=0.002)$

Test for overal $\mathrm{Cn}=2 . \mathrm{A}=3.08(P=0.002)$

$\begin{array}{rrrrr}20 & 0 & 3 & 8.2 \% & 3.14[0.14,69.95] \\ 20 & 6 & 20 & 50.6 \% & 1.56[0.42,576]\end{array}$

$\begin{array}{rrrrrrr}4 & 10 & 0 & 7 & 40.6 \% & 10.38[0.47 .232,5.76\end{array}$

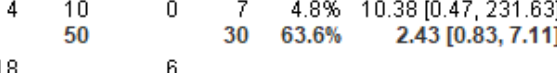

$\begin{array}{llllll}4 & 54 & 1 & 60 & 123 \% & 472[051,4361\end{array}$

$\begin{array}{rrrrrrr}10 & 94 & 2 & 102 & 24.1 \% & 5.95[1.27,27.92] \\ & 148 & & 162 & 36.4 \% & 5.54[1.56,19.67]\end{array}$

$14 \quad 3 \quad \begin{array}{llll}162 & 36.4 \% & 5.54[1.56,19.67]\end{array}$

18

$192 \quad 100.0 \% \quad 3.56[1.59,7.98]$

Tester

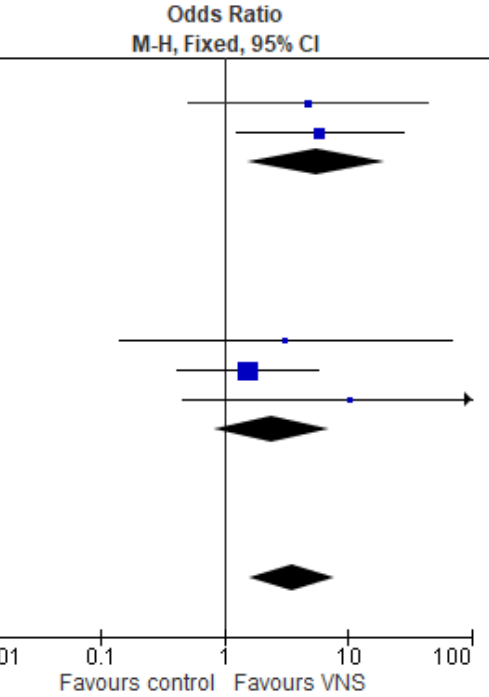


VNS Control

Odds Ratio

Odds Ratio

Study or Subgroup

nts Total Events Total Weight M-H, Fixed, 95\% C M-H. Fixed, $95 \% \mathrm{Cl}$

E03 (3.5 months) [RCT: High VNS ws Iow VNS]

E05 $3.4-4.5 \mathrm{~m}$

Subtotal $(95 \% \mathrm{Cl})$

$\begin{array}{llllll}54 & 0 & 60 & \text { Not estimable }\end{array}$

$\begin{array}{rrrrrr}1 & 94 & 0 & 102 & 3.6 \% & 3.29[0.13,81.73] \\ & 148 & & 162 & 3.6 \% & 3.29[0.13,81.73]\end{array}$

Total events

0

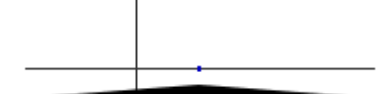

9.1.3 Comparative observational studies (adults)

Boon 2002 (3-4 months) [obs: VNS vS AED]

Gonen 2015 (4-5.7 yearden 2000 (3-3 lobs) [obs: VNS VS AED

Hoppe 2013 (80.4 months) [obs: WNS vS AEDS]

Subtotal $(95 \% \mathrm{Cl})$

Total events

Teterogenetty. $\mathrm{Chi}^{2}=6.47, \mathrm{df}=3(\mathrm{P}=0.09) \mathrm{F}^{2}=54$

test for

Total $(95 \% \mathrm{Cl})$

$\begin{array}{llllll}6 & 25 & 1 & 24 & 5.9 \% & 7.26[0.80,65.71]\end{array}$

$\begin{array}{llllll}20 & 2 & 20 & 14.5 \% & 0.31[0.06,1.59 \\ 20 & 4 & 20 & 2.5 \% & 0.04 .569]\end{array}$

$\begin{array}{rrrrrr}20 & 4 & 20 & 28.9 \% & 0.21[0.02,2.08] \\ 98 & & & 111 & 96.4 \% & 0.73[0.31,1.71]\end{array}$

10

15

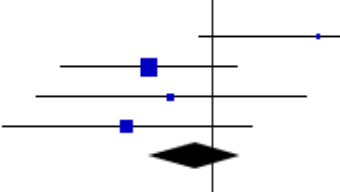

$273 \quad 100.0 \% \quad 0.82[0.37,1.84]$

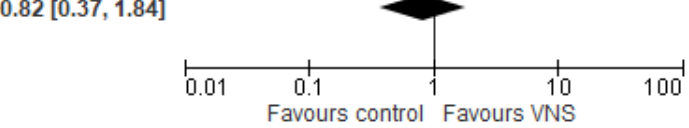

Heterogeneity: $\mathrm{Chi}^{2}=7.38, \mathrm{df}=4(\mathrm{P}=0.12) ; \mathrm{p}^{2}=46 \%$

$11 \quad 15$

Favours control 110 100 
Study or Subgroup

Experimental Control Mean Difference Mean Difference

E03 (3.5 months) [RCT: High VNS vs low VNS

(3.) V. Fixed, $95 \% \mathrm{Cl}$

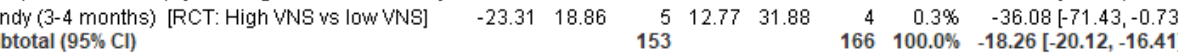

Heterogeneity: $C \mathrm{Cl}^{2}=2.12$, df $=2(P=0.35) ; I^{2}=6 \%$
Test for overall effect $Z=19.29(P<0.00001)$

8.1.3 Comparative observational studies (adults)

btotal $(95 \% \mathrm{Cl})$

0 Not estimable

Test for overarall effect: Not applica

Total $(95 \% \mathrm{Cl})$

$153 \quad 166 \quad 100.0 \%-18.26[-20.12,-16.41$

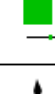

Teterogeneity: $C \mathrm{Ch}^{2}=2.12, \mathrm{df}=2(P=0.35) ; \mathrm{F}^{2}=6$

Test for subqroup differences: Not applicable

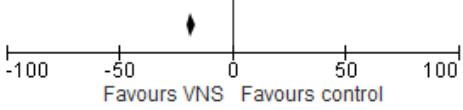




\begin{tabular}{|c|c|c|c|c|c|c|c|c|c|}
\hline \multirow{2}{*}{$\frac{\text { Study or Subgroup }}{\text { 31.1.1 RCTs (adults) }}$} & \multicolumn{2}{|c|}{ VNS } & \multicolumn{2}{|c|}{ Control } & \multirow[t]{2}{*}{ Weight } & \multirow{2}{*}{$\begin{array}{c}\text { Risk Ratio } \\
\text { M-H, Fixed, } 95 \% \mathrm{Cl}\end{array}$} & & \multirow{2}{*}{\multicolumn{2}{|c|}{$\begin{array}{c}\text { Risk Ratio } \\
\text { M-H, Fixed, } 95 \% \mathrm{Cl}\end{array}$}} \\
\hline & & & & & & & & & \\
\hline $\begin{array}{l}\text { Pulse (12 months) [RCT: VNS vs BMP] } \\
\text { Subtotal }(95 \% \text { CI) }\end{array}$ & 3 & $\begin{array}{l}31 \\
31\end{array}$ & 11 & $\begin{array}{l}28 \\
28\end{array}$ & $\begin{array}{l}47.1 \% \\
47.1 \%\end{array}$ & $\begin{array}{l}0.25[0.08,0.79] \\
0.25[0.08,0.79]\end{array}$ & & & \\
\hline $\begin{array}{l}\text { Total events } \\
\text { Heterogeneity: Not applicable } \\
\text { Test for overall effect: } Z=2.35(P=0.02)\end{array}$ & 3 & & 11 & & & & & & \\
\hline 31.1.3 Comparative observational studies (adults) & & & & & & & & & \\
\hline $\begin{array}{l}\text { Tatum } 2001(13.2 \text { months) [obs: VNS vs control] } \\
\text { Subtotal }(95 \% \mathrm{Cl})\end{array}$ & 4 & $\begin{array}{l}21 \\
21\end{array}$ & 13 & $\begin{array}{l}21 \\
21\end{array}$ & $\begin{array}{l}52.9 \% \\
52.9 \%\end{array}$ & $\begin{array}{l}0.31[0.12,0.79] \\
0.31[0.12,0.79]\end{array}$ & & & \\
\hline $\begin{array}{l}\text { Total events } \\
\text { Heterogeneity: Not applicable } \\
\text { Test for overall effect: } Z=2.45(P=0.01)\end{array}$ & 4 & & 13 & & & & & & \\
\hline Total $(95 \% \mathrm{Cl})$ & & 52 & & 49 & $100.0 \%$ & $0.28[0.13,0.58]$ & & & \\
\hline Total events & 7 & & 24 & & & & & & \\
\hline $\begin{array}{l}\text { Heterogeneity: Chi } \mathrm{i}^{2}=0.08, \mathrm{df}=1(\mathrm{P}=0.77) ; \mathrm{l}^{2}=0 \% \\
\text { Test for overall effect: } Z=3.40(P=0.0007) \\
\text { Test for subqroup differences: } C \mathrm{Ch}^{\mathrm{z}}=0.08, \mathrm{df}=1(\mathrm{P}\end{array}$ & & & & & & & 0.01 & $\begin{array}{l}0.1 \\
\text { Favours VNS }\end{array}$ & $\begin{array}{l}1 \\
1 \\
\text { Favours control } \\
\end{array}$ \\
\hline
\end{tabular}

Total events

24 
Fig. 8

Study or Subgroup

4.1.1 RCTs (adults)

E05 (3.4-4.5 months) [RCT: High VNS vs low VNS] Pulse (12 months) [RCT: VNS vs BMP]

Subtotal $(95 \% \mathrm{Cl})$

Total events

Heterogeneity: $\mathrm{Chi}^{2}=0.02, \mathrm{df}=1(P=0.89) ; \mathrm{I}^{2}=0 \%$ Test for overall effect: $Z=0.57(P=0.57)$

4.1.3 Comparative observational studies (adults) Subtotal $(95 \% \mathrm{Cl})$

Total events

Heterogeneity: Not applicable

Test for overall effect: Not applicable

Total $(95 \% \mathrm{Cl})$

Total events

Heterogeneity: $\mathrm{Chi}^{2}=0.02, \mathrm{df}=1(\mathrm{P}=0.89) ; \mathrm{I}^{2}=0 \%$

Test for overall effect: $Z=0.57(P=0.57)$

Test for subgroup differences: Not applicable

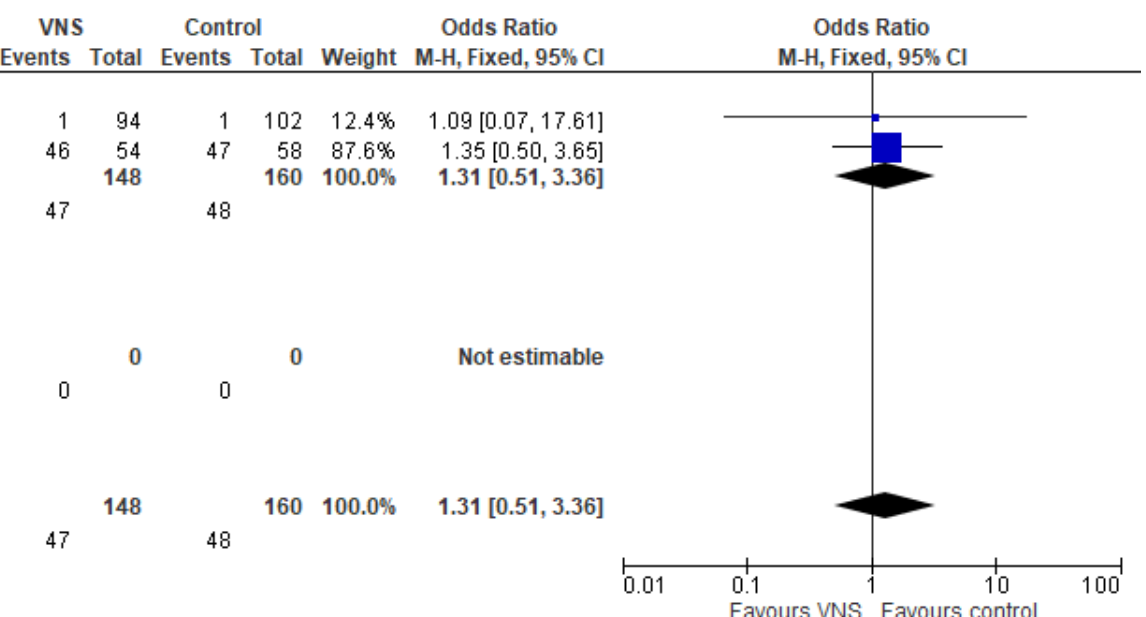


Study or Subgroup

Pulse (12 months) [RCT: VNS vs BMP] Subtotal (95\% CI)

Total events

Heterogeneity Not applicable

Test for overall effect: $Z=0.83(P=0.41)$

6.1.3 Comparative observational studies (adults)

Subtotal $(95 \% \mathrm{Cl})$

Total events

eterogeneity: Not applicable

Test for overall effect: Not applicable

Total $(95 \% \mathrm{Cl})$

Total events

eterogeneity: Not applicable

Test for overall effect $Z=0.83(P=0.41)$

Test for subaroup differences: Not applicable

5
VNS Control Odds Ratio $\quad$ Odds Ratio

Events Total Events Total Weight M- Odds Ratio

$\begin{array}{lllll}54 & 3 & 58 & 100.0 \% & 1.87[0.42,8.24] \\ 54 & & 58 & 100.0 \% & 1.87[0.42,8.24]\end{array}$ M-H, Fixed, $95 \%$ C

$\longrightarrow$

0

Not estimable

$\begin{array}{llll}54 & 58 & 100.0 \% & 1.87\end{array}[0.42,8.24]$

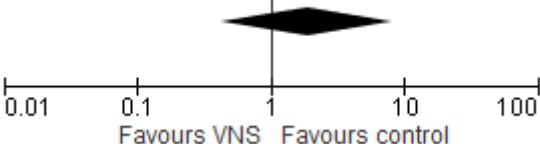




\section{Supplementary Files}

This is a list of supplementary files associated with this preprint. Click to download.

- Supplementarymaterials.docx 